\title{
Inversão do campo de onda completo (FWI) ao modelo conceitual do Campo de Búzios
}

\author{
Felipe Timóteo da Costa ${ }^{1}$, Ammir Ayman Karsou ${ }^{1}$, Ruann Carlos Mendanha da Silva ${ }^{1}$, Flavio Costa de Mesquita ${ }^{1}$, Marco
} Antonio Cetale Santos ${ }^{1}$, Roger Matsumoto Moreira ${ }^{1}$

${ }^{1}$ GISIS-UFF

Copyright 2019, SBGf - Sociedade Brasileira de Geofísica.

This paper was prepared for presentation at the $16^{\text {th }}$ International Congress of the Brazilian Geophysical Society, held in Rio de Janeiro, Brazil, August 19-22, 2019.

Contents of this paper were reviewed by the Technical Committee of the $16^{\text {th }}$ International Congress of The Brazilian Geophysical Society and do not necessarily represent any position of the SBGf, its officers or members. Electronic reproduction or storage of any part of this paper for commercial purposes without the written consent of The Brazilian Geophysical Society is prohibited.

\section{Resumo}

Os algoritmos de inversão usando o campo de onda completo (FWI) vêm se estabelecendo como importante técnica para a construção de modelos de velocidade de alta resolução. Entretanto, sua aplicação ainda apresenta diversos desafios e precisam ser avaliadas de acordo com o contexto geológico a ser estudado. Portanto, nesse trabalho foi construído um modelo conceitual simplificado baseado nas informações do Campo de Búzios na Bacia de Santos, e aplicada a FWI para avaliar se o algoritmo é capaz de recuperar as estruturas geologicamente complexas presentes no campo. Foram utilizados os dados sísmicos e os perfis de poço para a construção do modelo conceitual, e a inversão utilizou a equação da onda acústica, o método de otimização L-BFGS e o método do estado adjunto. $O$ modelo estimado recuperou as principais feições construídas a partir de um modelo inicial que simula um modelo construído pela tomografia. A qualidade do modelo estimado foi quantificada através da diferença RMS, que foi reduzida em aproximadamente $80 \%$ do modelo inicial

\section{Introdução}

Nos últimos anos a inversão do campo de onda completo (full waveform inversion - FWI) (LAILLY, 1983; TARANTOLA, 1984) vêm se tornando um dos principais métodos de construção de modelos de velocidade, pois os resultados alcançados com esse método alcançam modelos de propriedade de alta resolução. Entretanto, aplicação dos métodos de inversão e imageamento sísmico em contextos geológicos complexos, como por exemplo os reservatório do Pré-Sal na Bacia de Santos, ainda permanece um desafio para a indústria do petróleo (SHEN et al., 2018). As regiões abaixo das espessas camadas de sal são difíceis de estimar as propriedades físicas devido a grande profundidade, baixa iluminação e limitado conteúdo de frequência (VIRIEUX; OPERTO, 2009).

A validação dos algoritmos de inversão é uma etapa crucial para a aplicação em dados reais. Uma abordagem típica é a construção de modelos conceituais que representam a geologia da região estudada, como por exemplo o modelo Marmousi (VERSTEEG, 1994). Como os resultados do método de inversão fornecem respostas distintas de acordo com a região de estudo, é importante aplicá-los direcionados a área de interesse. Assim, a construção de modelos conceituais que representem as principais dificuldades encontradas na região de estudo, como por exemplo, as espessas camadas de sal estratificado, grandes variações laterais de velocidades, falhas geológicas, entre outros, são importantes para a validação dos algoritmos de inversão.

Portanto, o objetivo deste trabalho é avaliar o comportamento do algoritmo de FWI desenvolvido pelo grupo aplicados ao contexto do pré-sal brasileiro utilizando as informações do campo de Búzios na Bacia de Santos para a construção de um modelo conceitual que melhor represente a geologia da região.

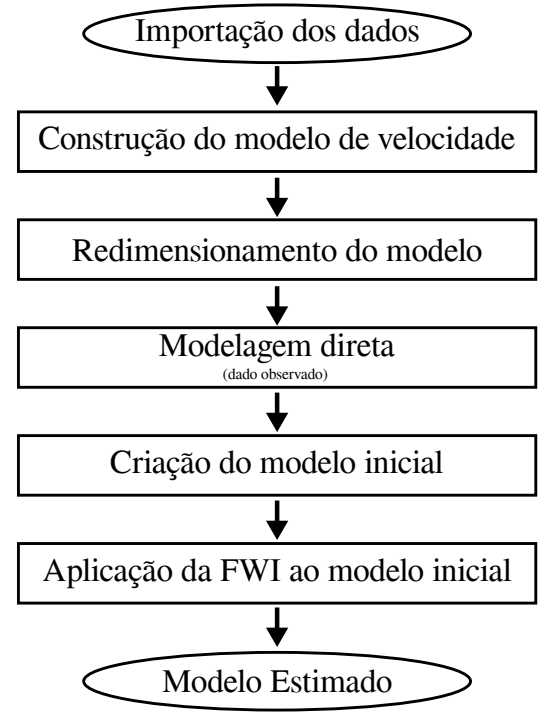

Figura 1: Fluxograma ilustrando a metodologia utilizada para a criação do modelo e inversão FWI.

\section{Materiais e Métodos}

A Figura 1. apresenta o fluxo de trabalho realizado nesse estudo. Inicialmente foram carregados os dados sísmicos pós empilhamento e o perfil sônico do campo de Búzios, em seguida, foram interpretados os horizontes sísmicos utilizando as informações dos marcadores dos perfis compostos. As informações dos perfis sônicos foram extrapoladas de modo a seguir a geometria dos horizontes interpretados. $\mathrm{O}$ modelo de velocidade conceitual foi gerado e posteriormente redimensionado. Uma modelagem sísmica foi realizada para gerar os dados 
observados. O modelo conceitual foi suavizado para simular o modelo de entrada para a inversão, e por fim aplicado o algoritmo de FWI para a geração do modelo estimado.

\section{Contexto Geológico}

A bacia de Santos vem se consolidando no cenário petrolífero nacional através da constante oferta de blocos exploratórios e de investimentos de pesquisa por parte de companhias nacionais e estrangeiras. Tradicionalmente, tais investimentos vêm sendo aplicados em fronteiras exploratórias em águas profundas e ultraprofundas, buscando reservatórios em arenitos do Cretáceo Superior. Recentemente, a seção pré-sal vem ganhando destaque devidos as grandes descobertas nos reservatórios carbonáticos. O Campo de Búzios está localizado na porção central da Bacia de Santos, a cerca de $180 \mathrm{~km}$ da costa do município do Rio de Janeiro em lâmina d'água de aproximadamente $1900 \mathrm{~m}$ de profundidade. Os reservatórios do Campo de Búzios se situam entre 5.000 e $6.0000 \mathrm{~m}$ de profundidade, e são representados pelas coquinas da $\mathrm{Fm}$. Itapema e os microbialitos da Fm. Barra Velha os quais estão sotopostos a espessa camada evaporítica referente a Fm. Ariri, composta majoritariamente por Halita tendo uma espessura variando da ordem de centenas de metros até mais de $2 \mathrm{~km}$ (ANP, 2016).

\section{Construção do modelo de velocidades conceitual}

Para a construção do primeiro modelo conceitual baseado nas informações do campo de Búzios foram utilizados o cubo de dados sísmicos 3D pós empilhamento migrado em profundidade e os perfis sônicos de quatro poços dentro da região do levantamento. A interpretação dos dados sísmicos foi realizada ao longo de todo o cubo sísmico, e foram considerados apenas quatro horizontes principais: Formação Marambaia (fundo marinho), Formação Ariri (topo do sal), Discordância Pré-Alagoas (base do sal) e a Formação Camboriú (embasamento econômico). Todos os horizontes são bem definidos na sísmica, com exceção da Formação Camboriú, que foi mapeada com a ajuda do atributo sísmico TecVa (BULHÕES; AMORIM, 2005). Todos os marcadores foram localizados a partir do perfil composto de cada poço, que foram usados para a interpretação sísmica ao longo de todo o cubo sísmico, gerando as principais geometrias do modelo geológico utilizado na construção do modelo de velocidade conceitual.

A figura 2 apresenta a seção selecionada para a construção do modelo conceitual 2D do campo de Búzios. A ausência de dados na região central da seção foi provocada por uma falha no arquivo de dados sísmicos, nessa região os horizontes foram interpolados. Entretanto, novos dados já foram solicitados para solucionar esse problema, assim os modelos que serão desenvolvidos em trabalhos futuros não sofrerão com o mesmo problema.

Após a interpretação dos horizontes, foi feita a extrapolação dos dados do perfil sônico de modo que os valores de velocidades respeitassem a geometria dos horizontes interpretados. As regiões com profundidades maiores que $7000 \mathrm{~m}$ foram ignoradas com o objetivo de reduzir o número total de amostras do modelo conceitual. Assumiu-se que o embasamento econômico

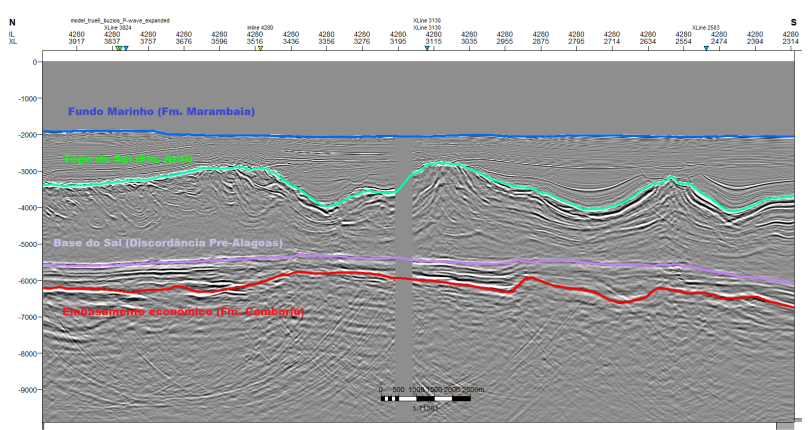

Figura 2: Seção sísmica e os horizontes interpretados das principais formações utilizada para criação do modelo geológico conceitual do campo de Buzios. O horizonte azul é o fundo marinho ( $F m$. Marambaia), em verde é o topo do sal (Fm. Ariri), o horizonte roxo é a Discordância PréAlagoas e o horizonte vermelho é a Fm. Camboriú.

possui velocidade constante igual à última amostra do perfil sônico, igual à $5668 \mathrm{~m} / \mathrm{s}$. O modelo gerado possui um espaçamento de malha de $5 \mathrm{~m}$ e 1735 amostras na horizontal e 1341 amostras na vertical. Para diminuir o custo computacional do cálculo do problema direto e inverso, o modelo conceitual foi reescalado e redimensionado de modo a reduzir o número de amostras. As dimensões finais do modelo conceitual foram de 433 amostras na horizontal e 326 amostras na vertical com um espaçamento de malha de $10 \mathrm{~m}$.

\section{Modelagem Acústica}

A equação da onda, núcleo da inversão, permite a estimativa do campo de pressão na superfície (dados calculados), e posteriormente, a comparação entre os dados observados obtendo assim o resíduo que será usado para calcular a função objetivo, que por sua vez, será minimizada iterativamente pelo método de otimização não linear. Em um meio acústico, isotrópico e heterogêneo com densidade constante, a equação da onda têm a seguinte expressão:

$$
\frac{1}{c^{2}(\mathbf{x})} \frac{\partial^{2}}{\partial t^{2}} p(\mathbf{x}, t)-\nabla^{2} p(\mathbf{x}, t)=w\left(\mathbf{x}_{s}, t\right),
$$

onde o vetor $\mathbf{x}$ representa as coordenadas espaciais (no caso bidimensional, $x \mathrm{e} \mathrm{z}$ ), $t$ o tempo, $c$ a velocidade de propagação da onda no meio, $p$ o campo de pressão e $w$ a assinatura da fonte. A aproximação por diferenças finitas (LEVANDER, 1989) foi utilizada para solucionar a equação da onda acústica numericamente e reproduzir o experimento sísmico.

\section{Método de otimização L-BFGS e Método adjunto}

A função objetivo usando a norma $\mathrm{L} 2$ mede a similaridade entre os dados observados e calculados, e possui a seguinte expressão para o caso sísmico:

$$
\chi(\mathbf{m})=\frac{1}{2} \int_{T} \int_{\partial \Omega_{s}} \int_{\partial \Omega_{r}}\left|\left(\mathbf{d}_{c a l}\left(\mathbf{x}_{r}, \mathbf{x}_{s}, t\right)\right)-\mathbf{d}_{o b s}\left(\mathbf{x}_{r}, \mathbf{x}_{s}, t\right)\right|^{2} d \mathbf{x}_{r} d \mathbf{x}_{s} d t,
$$

onde $\mathbf{x}_{r}, \mathbf{x}_{s}$ e $t$ são, respectivamente, as posições das estações receptoras, as posições das fontes e os instantes de tempo. E $\partial \Omega_{r}$ é o domínio que contém as posições 
das estações receptoras organizadas por tiro, $\partial \Omega_{s}$ o domínio que contém as posições das fontes, e $T$ o período de registro por tiro. Os termos $\mathbf{d}_{o b s}$ e $\mathbf{d}_{c a l}$ são os dados observados e calculados sendo o termo $\frac{1}{2}$ é uma conveniência matemática.

De acordo com Nocedal e Wright (2006), devido à não linearidade do problema sísmico e a grande quantidade de parâmetros, o modelo de velocidades deve ser estimado iterativamente utilizando um método de otimização local baseado no gradiente. Tais métodos garantem apenas a convergência para um mínimo local da função objetivo, por isso é fundamental que o modelo inicial esteja próximo ao mínimo global.

O método quasi-Newton L-BFGS vem sendo usado amplamente na resolução da FWI (MÉTIVIER et al., 2012). Entre os métodos baseados no gradiente, ele é o mais indicado devido à sua taxa de convergência supra linear. Além disso, ele utiliza uma aproximação da matriz Hessiana que é obtida através dos $k$-ésimos gradientes das iterações anteriores, calculados por diferenças finitas. Esse cálculo é feito iterativamente, de modo que a inversa da matriz Hessiana é atualizada a cada iteração (MÉTIVIER; BROSSIER, 2016). Logo, a equação de atualização do modelo de propriedades fica:

$$
\mathbf{m}_{k+1}=\mathbf{m}_{k}-\alpha_{k} H_{k} \nabla \chi\left(\mathbf{m}_{k}\right)
$$

onde $\alpha_{k}$ é o tamanho do passo e $H_{k}$ é a aproximação da inversa da matriz Hessiana e o produto $-H_{k} \nabla \chi\left(\mathbf{m}_{k}\right)$ é a direção de atualização. Uma vez definida a direção de atualização, é necessário definir o tamanho do passo $\alpha_{k}$. O tamanho do passo deve garantir que, para uma dada direção de atualização a função objetivo seja mínima. Esse critério pode ser alcançado utilizando as condições de Wolfe: (1) O passo deve ser calculado, de modo que haja uma diminuição suficiente da função objetivo para uma dada direção; (2) O passo não deve ser pequeno, evitando atualizações insignificantes. Além da definição do tamanho do passo, é possível a utilização de uma restrição garantindo que o modelo atualizado esteja dentro de um domínio plausível (MÉTIVIER; BROSSIER, 2016).

Desenvolvido inicialmente por Lailly (1983) e Tarantola (1984) o método do estado adjunto determina a direção de atualização de forma eficiente evitando o cálculo das derivadas de Fréchet. No caso acústico, onde o parâmetro estimado é o modelo de velocidade $(\mathbf{m}=c(\mathbf{x}))$, o gradiente da função objetivo para um único tiro possui a seguinte forma (FICHTNER, 2010):

$$
\nabla \chi(c)=\frac{2}{c^{3}(\mathbf{x})} \int_{T} \int_{D} p^{\dagger}(\mathbf{x}, t)\left[\frac{\partial^{2} p(\mathbf{x}, t)}{\partial t^{2}}\right] d \mathbf{x} d t,
$$

sendo que as integrais são avaliadas para todos instantes de tempo e para todo o domínio computacional. A $2^{\mathrm{a}}$ derivada do campo de pressão é obtida através da modelagem direta utilizando a equação 1. E o campo adjunto, $p^{\dagger}(\mathbf{x}, t)$, é obtido através da modelagem reversa no tempo, utilizando as condições finais e a fonte adjunta (FICHTNER et al., 2008):

$$
\frac{1}{c^{2}(\mathbf{x})} \frac{\partial^{2}}{\partial t^{2}} p^{\dagger}(\mathbf{x}, t)-\nabla^{2} p^{\dagger}(\mathbf{x}, t)=\frac{\partial \chi}{\partial p}
$$

no caso da norma L2 (equação 2), a fonte adjunta é dada pelo resíduo: diferença entre o dado observado e o calculado, $\Delta \mathbf{d}=\mathbf{d}_{c a l}\left(\mathbf{x}_{r}, \mathbf{x}_{s}, t\right)-\mathbf{d}_{o b s}\left(\mathbf{x}_{r}, \mathbf{x}_{s}, t\right)$. Como é possível notar, os operadores utilizados para o cálculo do campo de pressão e campo adjunto são os mesmos com exceção do termo fonte. O cálculo de $\nabla \chi(c)$ é muito semelhante ao método de Migração Reversa no Tempo (Reverse Time Migration - RTM), menos as constantes e o fator de derivada temporal (TARANTOLA, 1984).

\section{Resultados}

\section{Modelo conceitual}

O modelo conceitual baseado nos dados sísmicos e nos perfis sônicos do Campo de Búzios é apresentado na Figura 3. Os perfis de poços não possuíam informações em todo pacote sedimentar do pós-sal, por isso, adotou-se uma velocidade constante, de $1900 \mathrm{~m} / \mathrm{s}$, na região sem as informações do perfil sônico. O modelo conceitual simula os espessos corpos salinos presentes na Bacia de Santos incluindo as estratificações provocadas pela presença de sais com velocidades diferentes da Halita, como por exemplo Anidrita, Gipsita, Taquidrita, Carnalita e Silvinita. Os reservatórios carbonáticos abaixo da Sequência Evaporítica também foram representados, assim como o embasamento econômico.

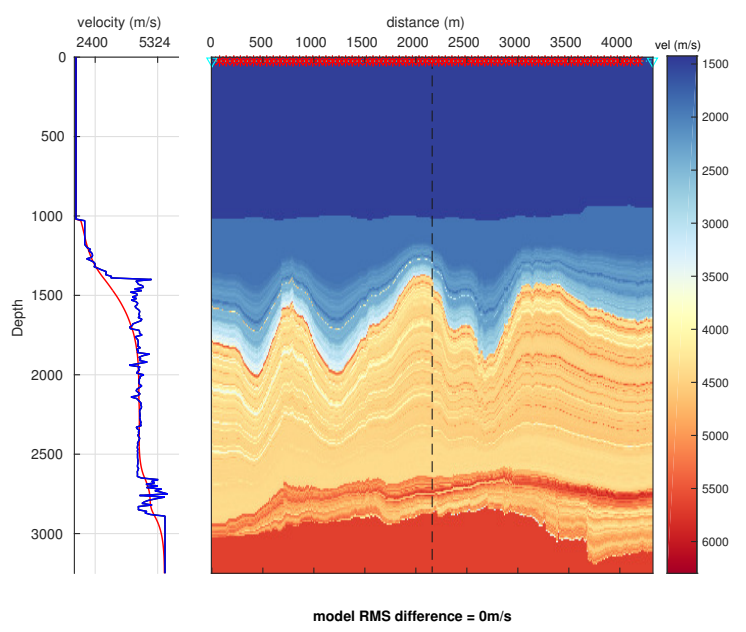

Figura 3: Modelo conceitual 2D para o campo de Búzios (bacia de Santos) gerado através dos dados sísmicos pós empilhamento e dos perfis sônicos. A linha pontilhada indica a posição do perfil lateral, que compara as velocidades do modelo conceitual e inicial.

\section{Modelo inicial}

Para aplicação da FWI é necessário um modelo de velocidade inicial que esteja próximo do modelo verdadeiro para garantir que o método de otimização convirja para um mínimo global. Isso normalmente é alcançado através de técnicas tomográficas quando utilizado dados reais. Nesse caso, o modelo inicial foi obtido através da suavização do modelo conceitual (crime de inversão), simulando um modelo obtido pela tomografia. Essa abordagem é tipicamente realizada para avaliar os resultados alcançados pelo algoritmo de inversão (WIRGIN, 2004). 


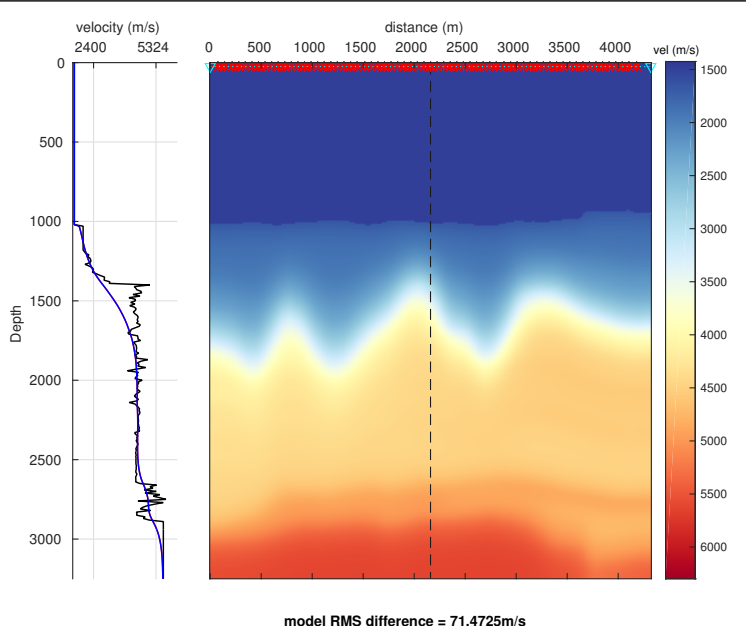

(a)

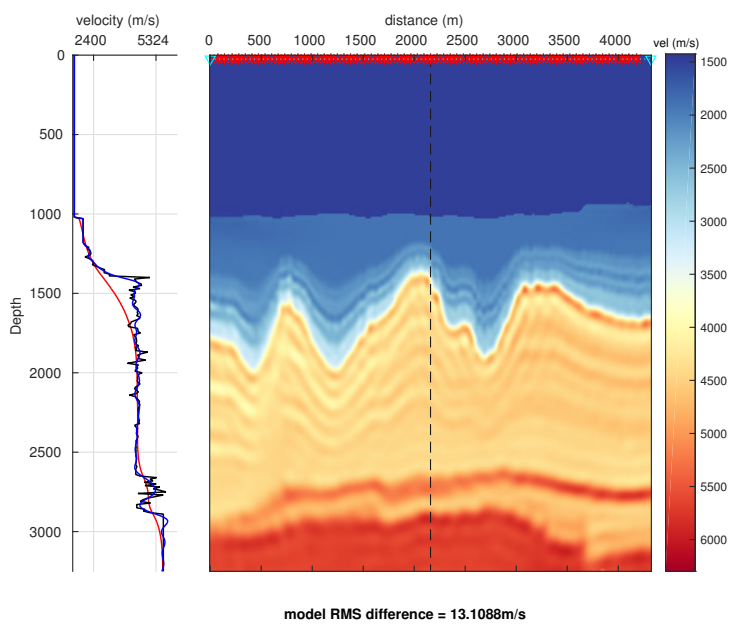

(b)

Figura 4: a) Modelo inicial utilizado nos algoritmos de modelagem e inversão sísmica. Esse modelo foi construído através da suavização do modelo conceitual. b) Modelo de velocidade estimado pela inversão FWI. A linha pontilhada indica a posição do perfil lateral.

\section{Modelo estimado}

Os dados observados foram gerados sinteticamente utilizando o modelo conceitual do campo de Búzios (Figura 3) utilizando uma wavelet Ricker (RYAN, 1994), a máxima frequência de corte foi $30 \mathrm{~Hz}$. Foram realizados 64 tiros (indicados com asteriscos vermelhos nos modelos) com tempo de registro de $4 \mathrm{~s}$. Os receptores (indicadas as posições iniciais e finais por triângulos azuis nos modelos) foram posicionados na superfície em cada ponto da malha de modelagem.

Para aplicação da FWI foi utilizada a abordagem multiescala no domínio da frequência (BUNKS et al., 1995), foram utilizadas as frequências $0-12 \mathrm{~Hz}, 0$ $15 \mathrm{~Hz}, \quad 0-18 \mathrm{~Hz}, \quad 0-21 \mathrm{~Hz}, \quad 0-24 \mathrm{~Hz}, \quad 0-27 \mathrm{~Hz}$ e $0-30 \mathrm{~Hz}$. Os dados observados e calculados foram gerados utilizando as mesmas wavelets para evitar as etapas de precondicionamento dos dados e estimativa da wavelet a partir dos dados observados.
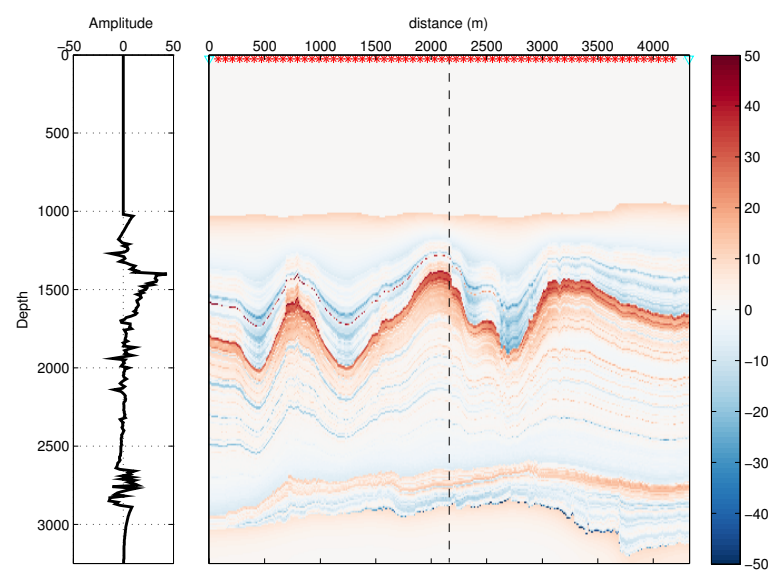

Relative Difference Velocity Initial Model [True - Initial ] - (\%)

(a)
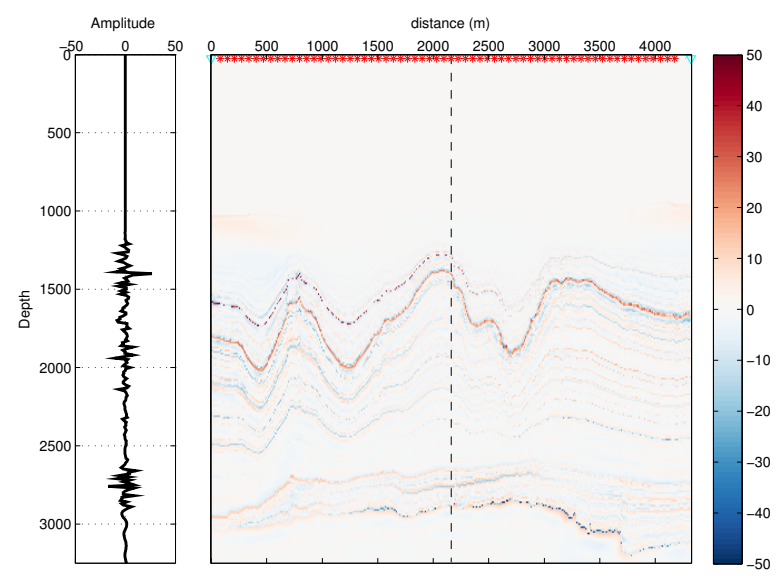

Relative Difference Velocity Inverted Model [True - Inverted ] - (\%)

(b)

Figura 5: a) Diferença relativa entre o modelo conceitual e o modelo inicial. b) Diferença relativa entre o modelo conceitual e o modelo estimado. A linha pontilhada indica a posição do perfil lateral.

O valor RMS da diferença entre o modelo conceitual e os modelos iniciais e estimados foram usados como medida da qualidade. A diferença RMS entre 0 modelo conceitual e inicial era de $71,4725 \mathrm{~m} / \mathrm{s}$, ao final do processo de inversão a diferença entre o modelo conceitual e estimado foi de $13,1088 \mathrm{~m} / \mathrm{s}$, uma redução de aproximadamente $80 \%$. As diferença ponto a ponto entre os modelos conceitual-inicial e conceitual-estimado podem ser observadas nas Figuras $5 a$ e 5b, respectivamente.

\section{Discussão e Conclusão}

O modelo de velocidade estimado pelo algoritmo de FWI (Figura 4a) recuperou as principais estruturas geológicas construídas no modelo conceitual do campo de Búzios (Figura 3). É possível identificar as estratificações da seção pós-sal, assim como parte das estratificações dentro da seção evaporítica. A geometria do reservatório carbonático na seção pré-sal foi parcialmente recuperada, incluindo algumas falhas simuladas no mesmo. 
A melhora do modelo estimado foi quantificada através da diferença RMS, que diminuiu consideravelmente após a aplicação da inversão. A comparação entre as diferenças relativas ponto-a-ponto (Figuras 5 a e 5 b,respectivamente) indicam que as diversas camadas presentes no modelo foram preenchidas com as velocidades corretas, entretanto, na região das interfaces as diferenças permaneceram altas.

Mesmo considerando as simplificações realizadas nesse experimento sintético, como por exemplo as simplificações na construção do modelo conceitual, a ausência de ruído no experimento sísmico, a geometria de aquisição pouco realista e os "crimes de inversão", os testes realizados nesse estudo apontam para a viabilidade da construção de modelos de velocidade de alta resolução utilizando os algoritmos de FWI orientados a geologia do Campo de Búzios.

As próximas etapas do estudo levarão em consideração a construção dos modelos conceituais de forma mais realista, aumentando a densidade de seções sísmicas interpretadas ao longo do cubo de dados sísmicos, realizando o mapeamento detalhado dos horizontes da seção do pós-sal e das falhas geológicas, além disso, a utilização da impedância acústica para gerar as estratificações da camada evaporítica (GOBATTO et al., 2016) e da região do reservatório que estejam mais coerentes com os dados sísmicos. As etapas de geração dos dados sintéticos também podem ser aprimoradas com a introdução de ruídos e uma geometria de aquisição mais realista.

\section{Agradecimentos}

Gostaríamos de agradecer ao grupo GISIS pelo suporte, a CAPES, a FEC e a UFF pelas bolsas de estudo. Além disso, as empresas Schumberger e CGG pela disponibilização das licenças acadêmicas dos softwares utilizados.

\section{Referências}

ANP. Plano de Desenvolvimento - Campo de Búzios. [S.I.], 2016.

BULHÕES, É. M.; AMORIM, W. N. d. Princípio da SismoCamada Elementar e sua aplicação à Técnica Volume de Amplitudes (tecVA). 9th International Congress of the Brazilian Geophysical Society, 2005.

BUNKS, C. et al. Multiscale seismic waveform inversion. Geophysics, Society of Exploration Geophysicists, v. 60, n. 5, p. 1457-1473, 1995.

FICHTNER, A. Full seismic waveform modelling and inversion. [S.I.]: Springer Science \& Business Media, 2010.

FICHTNER, A. et al. Theoretical background for continental- and global-scale full-waveform inversion in the time-frequency domain. Geophysical Journal International, v. 175, n. 2, p. 665-685, 2008.
GOBATTO, F. et al. Refining velocity model within the salt section in Santos Basin: An innovative workflow to include the existing stratification and its considerations. SEG International Exposition and 86th Annual Meeting, SEG 2016, v. 35, p. 5328-5333, 2016.

LAILLY, P. The Seismic Inverse Problem as a Sequence of Before Stack Migrations. Conference on Inverse Scattering, Theory and Applications, Society for Industrial and Applied Mathematics, $\mathrm{p}$. 206-220, 1983.

LEVANDER, A. Finite-difference forward modeling in seismology. Encyclopedia of Solid Earth Geophysics, p. 410-431, 011989.

MÉTIVIER, L.; BROSSIER, R. The seiscope optimization toolbox: A large-scale nonlinear optimization library based on reverse communicationthe seiscope optimization toolbox. Geophysics, GeoScienceWorld, v. 81, n. 2, p. F1-F15, 2016.

MÉTIVIER, L. et al. Optimization schemes for Full Waveform Inversion : the preconditioned truncated Newton method. SEG Annual Meeting, 2012.

NOCEDAL, J.; WRIGHT, S. Numerical Optimization. [S.I.]: Springer New York, 2006. (Springer Series in Operations Research and Financial Engineering). ISBN 9780387303031.

RYAN, H. Ricker, Ormsby; Klander, Butterworth -A Choice of wavelets. CSEG Recorder, p. 24-25, 1994.

SHEN, X. et al. Full-waveform inversion: The next leap forward in subsalt imaging. The Leading Edge, v. 37, n. 1, p. 67b1-67b6, 2018.

TARANTOLA, A. Inversion of seismic reflection data in the acoustic approximation. Geophysics, v. 49, n. 8, p. 1259-1266, 1984.

VERSTEEG, R. The Marmousi experience: Velocity model determination on a synthetic complex data set. The Leading Edge, v. 13, n. 9, p. 927-936, 1994.

VIRIEUX, J.; OPERTO, S. An overview of full-waveform inversion in exploration geophysics. Geophysics, v. 74, n. 6, p. WCC1, 2009.

WIRGIN, A. The inverse crime. 2004. 